\title{
On maximum number of decoherent histories
}

\author{
Lajos Diósi * \\ KFKI Research Institute for Particle and Nuclear Physics \\ H-1525 Budapest 114, POB 49, Hungary
}

bulletin board ref.: gr-qc/9409028

\begin{abstract}
It is shown that $N^{2}$ is the upper limit for the number of histories in a decohering family of $N$-state quantum system. Simple criterion is found for a family of $N^{2}$ fine grained decohering histories of Gell-Mann and Hartle to be identical with a family of Griffiths' consistent quantum trajectories.
\end{abstract}

*E-mail: diosi@rmki.kfki.hu 
Several versions of consistent history theories [1, 3, 2, 田, fl have been proposed earlier to incorporate the interpretation of quantum state into the basic equations of the formalism. Here we are going to prove that the maximum number of consistent histories is equal in both decoherent history and consistent quantum trajectory theories of Gell-Mann and Hartle [3], and of Griffiths [5], respectively. Further numerous algebraic properties have been explored independently by Dowker and Kent [6].

Let us consider a closed N-state quantum system whose state space is the Hilbert space $\mathcal{H}$ of $\mathrm{N}$ dimensions. Let us introduce a time-ordered sequence of events and the corresponding operator

$$
C_{\alpha} \equiv P_{\alpha_{n}}\left(t_{n}\right) \ldots P_{\alpha_{2}}\left(t_{2}\right) P_{\alpha_{1}}\left(t_{1}\right)
$$

where the $P_{\alpha_{k}}\left(t_{k}\right)$ 's form complete orthogonal sets of Hermitian projectors for each $k=0,1, \ldots, n$ in turn. Given the Heisenberg state $\rho$ of the system, the $C_{\alpha}$ 's are said to generate a family of decoherent histories [3] provided the so-called decoherence fuctional is diagonal:

$$
D(\alpha, \beta) \equiv \operatorname{tr}\left(C_{\beta}^{\dagger} C_{\alpha} \rho\right)=0 \quad \text { for all } \alpha \neq \beta .
$$

Its diagonal elements will be assigned to decoherent histories as their probabilities $p$ :

$$
p(\alpha)=D(\alpha, \alpha) .
$$

Griffiths' quantum trajectories [5 can be identified by a certain subset of fine grained [3] decoherent histories. Accordingly, all events in the sequence (1) will be described by 1-dimensional (pure state) projectors. Griffiths assignes probabilities to his quantum trajectories without referring to the state $\rho$ and the decoherence functional either. Here, nevertheless, we show how Griffiths noninterference condition and his probability assignment can be formulated within the context of decoherent histories (1-3). To this end, assume that the decoherence condition (2) holds for a family of fine grained histories, i.e., the decoherence functional is diagonal. Griffiths noninterference condition imposes additional constraints on the nonzero diagonal elements. For any pair of initial and final labels $\alpha_{1}, \alpha_{n}$, at most one decoherent history $\alpha_{1}, \alpha_{2}, \ldots, \alpha_{k} \ldots, \alpha_{n}$ should make the corresponding diagonal element $D(\alpha, \alpha)$ nonvanishing. In other words, the endpoints $\alpha_{1}, \alpha_{n}$ should determine the whole string uniquely. The following factorization is then valid for nonzero diagonal elements:

$$
\begin{array}{r}
D(\alpha, \alpha)=\operatorname{tr}\left(P_{\alpha_{n}}\left(t_{n}\right) P_{\alpha_{n-1}}\left(t_{n-1}\right)\right) \operatorname{tr}\left(P_{\alpha_{n-1}}\left(t_{n-1}\right) P_{\alpha_{n-2}}\left(t_{n-2}\right)\right) \ldots \\
\ldots \times \operatorname{tr}\left(P_{\alpha_{2}}\left(t_{2}\right) P_{\alpha_{1}}\left(t_{1}\right)\right) \operatorname{tr}\left(P_{\alpha_{1}}\left(t_{n}\right) \rho\right) .
\end{array}
$$

Such family is considered consistent à la Griffiths, and assigned by the following probabilities:

$$
p^{G}(\alpha)=\frac{p(\alpha)}{p\left(\alpha_{1}\right)} .
$$


where $p\left(\alpha_{1}\right)=\operatorname{tr}\left(P_{\alpha_{1}}\left(t_{1}\right) \rho\right)$. From Eq. (4) it follows that Griffiths' probabilities $p^{G}(5)$ are independent of the Heisenberg state $\rho$ and depend only on the sequence (1) of events. Griffiths probability (5) of a given trajectory $\alpha$ is equal to the corresponding GMH probability (3) at the condition that the earliest event was $P_{\alpha_{1}}\left(t_{1}\right)$ with certainty; this condition relaxes the $\rho$-dependence of the Griffiths probabilities.

Since the total number of pairs $\alpha_{1}, \alpha_{n}$ is $N^{2}$, the maximum number of Griffiths' trajectories in a consistent family is $N^{2}$. In the generic case, the number of Griffiths' trajectories is equal to the number of pairs $\alpha_{1}, \alpha_{n}$ for which the overlap of the corresponding initial and final states is nonzero, i.e.:

$$
\operatorname{tr}\left(P_{\alpha_{n}}\left(t_{n}\right) P_{\alpha_{1}}\left(t_{1}\right)\right) \neq 0
$$

We show that $N^{2}$ is the upper limit for the number of decohering histories in GMH families, too. Let us start initially with pure state $\rho=|\psi\rangle\langle\psi|$ and introduce the unnormalized vectors

$$
\left|\varphi_{\alpha}\right\rangle=C_{\alpha}|\psi\rangle
$$

From the decoherence condition (2) it follows that they are orthogonal to each other:

$$
\left\langle\varphi_{\beta} \mid \varphi_{\alpha}\right\rangle=0 \quad \text { for all } \alpha \neq \beta
$$

The maximum number of (nonzero) orthogonal vectors in $\mathcal{H}$ is $N$.

Let us allow mixed states, too. Consider the orthogonal expansion $\rho=$ $\sum_{r=1}^{N} w_{r}\left|\psi_{r}\right\rangle\left\langle\psi_{r}\right|$ which is always possible with nonnegative normalized wights $w_{r}$. Consider the trivial embedding of our system into a larger one whose state space is $\mathcal{H} \otimes \mathcal{H}^{\prime}$ where $\mathcal{H}^{\prime}$ is also $N$-dimensional Hilbert space. Construct the following state vector in $\mathcal{H} \otimes \mathcal{H}^{\prime}$ :

$$
|\Psi\rangle=\sum_{r=1}^{N} \sqrt{w_{r}}\left|\psi_{r}\right\rangle \otimes\left|\psi_{r}^{\prime}\right\rangle
$$

where $\left\{\left|\psi_{r}^{\prime}\right\rangle, r=1, \ldots, N\right\}$ form a complete orthonormal system in $\mathcal{H}^{\prime}$. Introduce the following unnormalized vectors in $\mathcal{H} \otimes \mathcal{H}^{\prime}$ :

$$
\left|\Phi_{\alpha}\right\rangle=\left(C_{\alpha} \otimes \mathbf{1}\right)|\Psi\rangle .
$$

From Eqs. (7-10) one can prove that these vectors are also orthogonal to each other:

$$
\left\langle\Phi_{\beta} \mid \Phi_{\alpha}\right\rangle=\left\langle\varphi_{\beta} \mid \varphi_{\alpha}\right\rangle=0 \quad \text { for all } \alpha \neq \beta .
$$

In Hilbert space $\mathcal{H} \otimes \mathcal{H}^{\prime}$, the maximum number of orthogonal vectors is equal to the number $N \times N$ of dimensions. In such a way we have proven that the maximum number of histories in a given decohering family is $N^{2}$. (The general limit is $\operatorname{rank}(\rho) N$, as one could easily prove.) 
We see that the maximum number of consistent histories is identical at both GMH and Griffiths conditions. This fact makes one think about the extent of GMH decohering families are more general than Griffiths' ones. We are not yet able to present an exhaustive mathematical comparison. Nevertheless, we know the class of GMH families which is identical to Griffiths' families. If all initial events $P_{\alpha_{1}}\left(t_{1}\right)$ has nonvanishing overlaps (6) with all final events $P_{\alpha_{n}}\left(t_{n}\right)$ then a family of fine grained decoherent histories is a family of Griffiths' quantum trajectories.

The proof is rather easy. From the existence of all overlaps (6) it follows that, for each pair $\alpha_{1}, \alpha_{2}$ there must be at least one decoherent history $\alpha_{1}, \ldots, \alpha_{k}, \ldots, \alpha_{n}$ such that $D(\alpha, \alpha)$ is nonzero. [Otherwise $D(\alpha, \alpha)$ would be zero for all $\alpha_{2} \ldots \alpha_{n-1}$ and this would contradict to (6).] Such decoherent histories must exist for all $N^{2}$ different pairs $\alpha_{1}, \alpha_{n}$. Since the maximum number of decoherent histories, as shown above, is also $N^{2}$ there can be only a single one for each fixed pair $\alpha_{1}, \alpha_{n}$. This means that Griffiths' additional condition fulfils for the given family of $N^{2} \mathrm{GMH}$ decoherent histories.

For the generic fine grained decohering family when the number of positive overlaps (6) may be less than $N^{2}$ it is most likely that multiple histories will arise with the same endpoints $\alpha_{1}, \alpha_{n}$ and this denies the equivalence with any Griffiths family. We can not judge whether Griffiths' conditions are too restrictive or, oppositely, the GMH decoherence is too general. Nevertheless, GMH decoherence theory is formally equivalent with the von Neumann collapse theory [7]. Any restriction of the GMH decoherence might thus miss the probability assignment for certain families which are otherwise well-interpreted in the von Neumann language.

This work was supported by the grant OTKA No. 1822/1991.

\section{References}

[1] R.B. Griffiths, J. Stat. Phys. 36 (1984) 219.

[2] R. Omnès, Rev. Mod. Phys. 64 (1992) 339.

[3] M. Gell-Mann and J.B. Hartle, Phys. Rev. D 47 (1993) 3345.

[4] H.F. Dowker and J.J. Halliwell, Phys. Rev. D 46 (1992) 1580.

[5] R.B. Griffiths, Phys. Rev. Lett. 70 (1993) 2201.

[6] F. Dowker and A. Kent, preprint gr-qc/9409037; Cambridge preprint DAMTP/94-48.

[7] L. Diósi, Phys. Lett. 280 B (1992) 71. 